\title{
AOR
}

Selected Papers of \#AoIR2019:

The $20^{\text {th }}$ Annual Conference of the Association of Internet Researchers Brisbane, Australia / 2-5 October 2019

\section{TRUST UNDER TRIAL: \\ THE EFFECT OF SURGE PRICING ON TRUST TOWARD RIDE-HAILING PLATFORMS IN MANILA}

\author{
Godofredo Ramizo Jr \\ Oxford Internet Institute, University of Oxford \\ Introduction
}

Ride-hailing platforms such as Uber and Grab provide popular yet controversial transport alternatives to Manila, the Philippine's capital and a megacity with deficient public transport system, crowded city trains, and exploitative taxis. These ride-hailing platforms use algorithms which optimally match user demand and driver supply to provide origin-to-destination conveyance. This is made possible by an essential feature called surge pricing whereby standard fares are algorithmically increased by a multiplier during certain conditions, incentivising drivers to meet user demand. However, during direct experience of ride-hailing platforms in Metro Manila for 8 months in 2016, I glimpsed a puzzle: Many users distrust surge pricing since it results in higher fares, and yet this distrust over such an integral feature had minimal bearing on their persistently positive attitudes such as trust toward ride-hailing platforms.

Current literature on the link between digital platforms such as ride-hailing platforms and pro-technology attitudes such as trust generally focus on the Global North (Mittendorf 2017). The smaller number of empirical work on platform trust in the Global South (Soegoto 2018) does not yet explain why trust for platforms persists amid problematic platform features that may foment distrust. Hence, this paper investigates two related questions: First, how do attitudes on surge pricing influence trust toward Manila's ridehailing platforms? Second, why do attitudes on surge pricing influence trust of ridehailing platforms the way they do?

Suggested Citation (APA): Ramizo, G. (2019, October 2-5). Trust Under Trial: The Effect Of Surge Pricing On Trust Toward Ride-Hailing Platforms in Manila. Paper presented at AoIR 2019: The $20^{\text {th }}$ Annual Conference of the Association of Internet Researchers. Brisbane, Australia: AolR. Retrieved from http://spir.aoir.org. 
To accommodate various understandings of trust among study participants, this paper adopts the conventional usage of trust, defined by the Oxford Dictionary as a "firm belief in the reliability, truth or ability of someone or something."

\section{Methods}

I conducted in-depth semi-structured interviews with 30 respondents from Manila, who were gathered through purposive sampling from Facebook groups of ride-hailing users. Out of 133 interested participants, 22 were selected while maximising variability in home location, place of work, and frequency of use, among others. These 22 interviews and the 8 interviews from the pilot study were analysed through Nvivo using detailed eclectic coding.

\section{Findings and Analysis}

Out of 30 interviewees, 8 interviewees do not see issues in surge pricing as cause for concern. In contrast, 22 interviewees $(73 \%)$ showed distrust toward surge pricing because it causes exorbitant prices, its computation and triggers are not fully understood, and is unfairly triggered even when there is no traffic congestion. However, in the end, only two interviewees distrust ride-hailing apps due to their firm distrust of surge pricing. Everyone else have justifications which neutralise their negative perceptions of surge pricing, preventing any spillover toward their overall trust of ridehailing apps.

Reasons behind the persistence of trust can be categorized into four themes:

\section{Rational Choice Explanations}

Twenty-four respondents reason that their empirical experience indicates that using Manila's conventional transport modes would be much more difficult and timeconsuming. Hence, ride-hailing apps remain more advantageous despite any perceived injustice from surge pricing.

\section{Acceptance of Limited Transparency}

The presence of particular kinds of information in the app is enough to assuage 18 respondents even if the undisclosed information are needed to determine negative welfare impacts. Even if they note the algorithm's opacity, these respondents are satisfied just seeing the sum of surge price before booking because it sets their expectations. The issuance of receipts also reassures interviewees, even if the receipt only shows the total fare, and not the computation of all components.

This non-rational thinking reveals a cognitive bias: In contexts where users know information to be incomplete, these users overvalue the disclosed information and undervalue the undisclosed information despite having some awareness that the latter is necessary to gauge fairness and welfare impact. 


\section{Perceived Control}

Thirteen interviewees acknowledge the opaque computations and exorbitant costs as potential problems in surge pricing. Yet they view their choice to click the button to "book a ride" (or not) as "having control", which - in their view - nullifies any potential negative effect from prices that are perceived as unfair. For example, when asked whether the problems he sees in surge pricing are a big issue, an interviewee responds, "Not really. Because I can choose not to book." Likewise, respondents feel that the app does not try to "dictate" nor "overpower" them. It simply provides a choice, which can be ignored if unfavourable.

However, not choosing to press the "book a ride" button only removes from their gaze the potentially unfair prices, but does not eliminate the potential injustice in the underlying system. Again, this appears to be a cognitive bias where control of the superficial is perceived as control of the substantive, thereby giving users a false sense of control over potentially unjust situations.

\section{"That's how the app works": A Variant of System Trust}

For twenty-six interviewees, distrust of surge pricing was negated by justifications rooted in the ideational appeal of technological systems. Of these users, 16 explained that the use of automated algorithms justifies the suspension of doubt and the persistence of trust. For 22 respondents, while surge-pricing can appear unfair or exorbitant at first glance, it is part of a technological system with its own peculiar workings. It matters less to them whether surge pricing is transparent and fair, only that it is normal and therefore legitimate. For example, a respondent complains that his trip home could reach exorbitant surge prices, and yet he says, "Although... that's expected. That's how the app works. So, that's all."

This process is similar to what Luhmann (1989) calls system trust, which describes the tendency of people to possess an optimistic pro-system bias and assume that an impersonal system is predictably working and following formal safeguards. However there is a key difference here. In contrast to an optimistic pro-system bias, these interviewees believe that some undesirable experiences such as the risk of unfair surge pricing are also normal "parts of the system." Therefore, it is a bias that may legitimate valid problems in the technological system.

\section{Conclusion and Relevance}

Despite the impetus for distrust arising from surge pricing issues, trust for ride-hailing platforms persisted among respondents. Persistence of trust is partly due to users' rational calculations: Their experiences indicate that ride-hailing platforms provide net benefits despite the possibility of unfair surge pricing, especially when faced with the disagreeable alternative of using Manila's poor transport infrastructure. However, the persistence of trust was also due to cognitive biases among respondents, as reflected in constructs such as acceptance of limited transparency, perceived control, and a variant of system trust. Looking beyond the empirical context of Manila, the cognitive biases revealed by the data increase our understanding why trust in technological contexts 
may persist when besieged by distrust. The findings are also important because unscrupulous technology actors can use these cognitive biases to purposely instill trust on less than meritorious grounds, and hold a firm grip of users' trust even as they begin to harbor healthy skepticism over fairness and transparency.

\section{References}

Luhmann, N. 1989. Vertrauen. Ein Mechanismus Der Reduktion Sozialer Komplexitat. Stuttgart: Enke.

Mittendorf, Christoph. 2017. "The Implications of Trust in the Sharing Economy - An Empirical Analysis of Uber." In Proceedings of the 50th Hawaii International Conference on System Sciences, 10.

Soegoto, Herman. 2018. "Trust and Loyalty Level of Online Transportation Users." In . Atlantis Press. https://doi.org/10.2991/icobest-18.2018.12. 